\title{
Lattice structure of torsion classes for hereditary artin algebras.
}

\author{
Claus Michael Ringel
}

\begin{abstract}
Let $\Lambda$ be a connected hereditary artin algebra. We show that the set of functorially finite torsion classes of $\Lambda$-modules is a lattice if and only if $\Lambda$ is either representation-finite (thus a Dynkin algebra) or $\Lambda$ has only two simple modules. For the case of $\Lambda$ being the path algebra of a quiver, this result has recently been established by Iyama-ReitenThomas-Todorov and our proof follows closely their considerations.
\end{abstract}

Let $\Lambda$ be a connected hereditary artin algebra. The modules considered here are left $\Lambda$-modules of finite length, $\bmod \Lambda$ denotes the corresponding category. The subcategories of $\bmod \Lambda$ we deal with are always assumed to be closed under direct sums and direct summands (in particular closed under isomorphisms). In this setting, a subcategory is a torsion class (the class of torsion modules for what is called a torsion pair or a torsion theory) provided it is closed under factor modules and extensions. The torsion classes form a partially ordered set with respect to inclusion, it will be denoted by tors $\Lambda$. This poset clearly is a lattice (even a complete lattice). Auslander and Smalø have pointed out that a torsion class $\mathcal{C}$ in $\bmod \Lambda$ is functorially finite if and only if it has a cover (a cover for $\mathcal{C}$ is a module $C$ such that $\mathcal{C}$ is the set of modules generated by $C$ ), we denote by f-tors $\Lambda$ the set of functorially finite torsion classes in $\bmod \Lambda$.

In a recent paper [IRTT], Iyama, Reiten, Thomas and Todorov have discussed the question whether also the poset f-tors $\Lambda$ (with the inclusion order) is a lattice.

Theorem. The poset $\mathrm{f}$-tors $\Lambda$ is a lattice if and only if $\Lambda$ is representation finite or $\Lambda$ has precisely two simple modules.

Iyama, Reiten, Thomas, Todorov have shown this in the special case when $\Lambda$ is a $k$-algebra with $k$ an algebraically closed field (so that $\Lambda$ is Morita equivalent to the path algebra of a quiver). The aim of this note is to provide a proof in general. We follow closely the strategy of the paper [IRTT] and we will use Remark 1.13 of [IRTT] which asserts that a meet or a join of two elements $\mathcal{C}_{1}, \mathcal{C}_{2}$ in f-tors $\Lambda$ exists if and only if the meet or the join of $\mathcal{C}_{1}, \mathcal{C}_{2}$ formed in tors $\Lambda$ belongs to f-tors $\Lambda$, respectively.

\section{Normalization.}

Let $\mathcal{X}$ be a class of modules. We denote by $\operatorname{add}(\mathcal{X})$ the modules which are direct summands of direct sums of modules in $\mathcal{X}$. A module $M$ is generated by $\mathcal{X}$ provided $M$ is a factor module of a module in $\operatorname{add}(\mathcal{X})$, and $M$ is cogenerated by $\mathcal{X}$ provided $M$ is a submodule of a module in $\operatorname{add}(\mathcal{X})$. The subcategory of all modules generated by $\mathcal{X}$ is denoted by $\mathcal{G}(\mathcal{X})$. In case $\mathcal{X}=\{X\}$ or $\mathcal{X}=\operatorname{add} X$, we write $\mathcal{G}(X)$ instead of $\mathcal{G}(\mathcal{X})$, and

2010 Mathematics Subject Classification. Primary 16G10, 18E40. Secondary: 05E10, 16D90, 16G70. 
use the same convention in similar situations. We write $\mathcal{T}(X)$ for the smallest torsion class containing the module $X$ (it is the intersection of all torsion classes containing $X$, and it can be constructed as the closure of $\{X\}$ using factor modules and extensions).

Since $\Lambda$ is assumed to be hereditary, we write $\operatorname{Ext}(X, Y)$ instead of $\operatorname{Ext}^{1}(X, Y)$. Recall that a module $X$ is said to be exceptional provided it is indecomposable and has no selfextensions (this means that $\operatorname{Ext}(X, X)=0$ ).

Following Roiter [Ro], we say that a module $M$ is normal provided there is no proper direct decomposition $M=M^{\prime} \oplus M^{\prime \prime}$ such that $M^{\prime}$ generates $M^{\prime \prime}$ (this means: if $M=$ $M^{\prime} \oplus M^{\prime \prime}$ and $M^{\prime}$ generates $M^{\prime \prime}$, then $\left.M^{\prime \prime}=0\right)$. Of course, given a module $M$, there is a direct decomposition $M=M^{\prime} \oplus M^{\prime \prime}$ such that $M^{\prime}$ is normal and $M^{\prime}$ generates $M^{\prime \prime}$ and one can show that $M^{\prime}$ is determined by $M$ uniquely up to isomorphism, thus we call $M^{\prime}=\nu(M)$ a normalization of $M$. This was shown already by Roiter [Ro], and later by Auslander-Smalø [AS]. It is also a consequence of the following Lemma which will be needed for our further considerations.

Lemma 1. (a) Let $\left(f_{1}, \ldots, f_{t}, g\right): X \rightarrow X^{t} \oplus Y$ be an injective map for some natural number $t$, with all the maps $f_{i}$ in the radical of $\operatorname{End}(X)$. Then $X$ is cogenerated by $Y$.

(b) Let $\left(f_{1}, \ldots, f_{t}, g\right): X^{t} \oplus Y \rightarrow X$ be a surjective map for some natural number $t$, with all the maps $f_{i}$ in the radical of $\operatorname{End}(X)$, then $Y$ generates $X$.

Proof. (a) Assume that the radical $J$ of $\operatorname{End}(X)$ satisfies $J^{m}=0$. Let $W$ be the set of all compositions $w$ of at most $m-1$ maps of the form $f_{i}$ with $1 \leq i \leq t$ (including $\left.w=1_{X}\right)$. We claim that $(g w)_{w \in W}: X \rightarrow Y^{|W|}$ is injective. Take a non-zero element $x$ in $X$. Then there is $w \in W$ such that $w(x) \neq 0$ and $f_{i} w(x)=0$ for $1 \leq i \leq t$. Since $\left(f_{1}, \ldots, f_{t}, g\right)$ in injective and $w(x) \neq 0$, we have $\left(f_{1}, \ldots, f_{t}, g\right)(w(x)) \neq 0$. But $f_{i} w(x)=0$ for $1 \leq i \leq t$, thus $g(w(x)) \neq 0$. This completes the proof.

(b) This follows by duality.

Corollary (Uniqueness of normalization). Let $M$ be a module. Assume that $M=M_{0} \oplus M_{1}=M_{0}^{\prime} \oplus M_{1}^{\prime}$ such that both $M_{0}$ and $M_{0}^{\prime}$ generate $M$. Then there is a module $N$ which is a direct summand of both $M_{0}$ and $M_{0}^{\prime}$ which generates $M$.

Proof: We may assume that $M$ is multiplicity free. Write $M_{0} \simeq N \oplus C, M_{0}^{\prime} \simeq N \oplus C^{\prime}$, such that $C, C^{\prime}$ have no indecomposable direct summand in common. Now, $N \oplus C$ generates $N \oplus C^{\prime}$ generates $N \oplus C$ generates $C$. We see that $N \oplus C$ generates $C$, such that the maps $C \rightarrow C$ used belong to the radical of $\operatorname{End}(C)$ ( since they factor through $\operatorname{add}\left(N \oplus C^{\prime}\right)$ and no indecomposable direct summand of $C$ belongs to add $\left.\left(N \oplus C^{\prime}\right)\right)$. Lemma 1 asserts that $N$ generates $C$, thus it generates $M$.

Proposition 1. If $T$ has no self-extensions, then $T$ is a cover for the torsion class $\mathcal{T}(T)$. Conversely, if $\mathcal{T}$ is a torsion class with cover $C$, then $\nu(C)$ has no self-extensions.

Proof. For the first assertion, one has to observe that $\mathcal{G}(T)$ is closed under extensions, thus equal to $\mathcal{T}(T)$. This is a standard result say in tilting theory. Here is the argument: let $g^{\prime}: T^{\prime} \rightarrow M^{\prime}$ and $g^{\prime \prime}: T^{\prime \prime} \rightarrow M^{\prime \prime}$ be surjective maps with $T^{\prime}, T^{\prime \prime}$ in add $T$. Let $0 \rightarrow$ $M^{\prime} \rightarrow M \rightarrow M^{\prime \prime} \rightarrow 0$ be an exact sequence. The induced exact sequence with respect to $g^{\prime \prime}$ is of the form $0 \rightarrow M^{\prime} \rightarrow Y_{1} \rightarrow T^{\prime \prime} \rightarrow 0$ with a surjective map $g_{1}: Y_{1} \rightarrow M$. Since $\Lambda$ is hereditary and $g^{\prime}$ is surjective, there is an exact sequence $0 \rightarrow T^{\prime} \rightarrow Y_{2} \rightarrow T^{\prime \prime} \rightarrow 0$ 
with a surjective map $g_{2}: Y_{2} \rightarrow Y_{1}$. Since $\operatorname{Ext}\left(T^{\prime \prime}, T^{\prime}\right)=0$, we see that $Y_{2}$ is isomorphic to $T^{\prime} \oplus T^{\prime \prime}$, thus in add $T$. And there is the surjective map $g_{1} g_{2}: Y_{2} \rightarrow M$.

For the converse, we may assume that $C$ is normal and have to show that $C$ has no self-extension. Let $C_{1}, C_{2}$ be indecomposable direct summands of $C$ and assume for the contrary that there is a non-split exact sequence

$$
0 \rightarrow C_{1} \rightarrow M \rightarrow C_{2} \rightarrow 0
$$

Now $M$ belongs to $\mathcal{T}$, thus it is generated by $C$, say there is a surjective map $C^{\prime} \rightarrow M$ with $C^{\prime} \in$ add $C$. Write $C^{\prime}=C_{2}^{t} \oplus C^{\prime \prime}$ such that $C_{2}$ is not a direct summand of $C^{\prime \prime}$. Consider the surjective map $C_{2}^{t} \oplus C^{\prime \prime} \rightarrow M \rightarrow C_{2}$. Since the last map $M \rightarrow C_{2}$ is not a split epimorphism, all the maps $C_{2} \rightarrow C_{2}$ involved belong to the radical of $\operatorname{End}\left(C_{2}\right)$. According to Lemma $1, C^{\prime \prime}$ generates $C_{2}$. This contradicts the assumption that $C$ is normal.

Remark. As we have mentioned, normal modules have been considered by Roiter, but actually, he used a slightly deviating name, calling them "normally indecomposable".

\section{Ext-cycles.}

An Ext-cycle of cardinality $t$ is a sequence $X_{1}, X_{2}, \ldots, X_{m}$ of pairwise orthogonal bricks such that $\operatorname{Ext}\left(X_{i-1}, X_{i}\right) \neq 0$ for $1 \leq i \leq m$, with $X_{0}=X_{m}$. An Ext-pair is an Ext-cycle of cardinality 2 consisting of exceptional modules. (One may call an Ext-cycle $X_{1}, \ldots, X_{m}$ minimal provided there is no Ext-cycle of smaller cardinality which uses (some of) these modules. Using this definition, the Ext-pairs are just the minimal Ext-cycles of cardinality 2.)

Proposition 2. If $X_{1}, X_{2}, \ldots, X_{m}$ is an Ext-cycle, then $\mathcal{T}\left(X_{1}, \ldots, X_{m}\right)$ has no cover.

Proof: Let $\mathcal{F}=\mathcal{F}\left(X_{1}, \ldots, X_{m}\right)$ be the extension closure of $X_{1}, \ldots, X_{m}$, thus the class of modules with a filtration with factors of the form $X_{i}$, where $1 \leq i \leq m$. According to [R], $\mathcal{F}$ is an abelian subcategory with exact embedding functor, with (relative) simple objects the modules $X_{1}, \ldots, X_{m}$. The objects in $\mathcal{F}$ have finite (relative) length, thus also the (relative) Loewy length for these objects is defined. We denote by $\mathcal{F}_{t}$ the full subcategory of objects in $\mathcal{F}$ of (relative) Loewy length at most $t$.

We have

$$
\mathcal{F}_{1} \subseteq \mathcal{F}_{2} \subseteq \cdots \subseteq \mathcal{F}_{t} \subseteq \cdots
$$

and therefore

$$
\mathcal{G}\left(\mathcal{F}_{1}\right) \subseteq \mathcal{G}\left(\mathcal{F}_{2}\right) \subseteq \cdots \subseteq \mathcal{G}\left(\mathcal{F}_{t}\right) \subseteq \cdots
$$

Let $\mathcal{G}=\bigcup_{t} \mathcal{G}\left(\mathcal{F}_{t}\right)$. We claim that $\mathcal{G}=\mathcal{T}\left(X_{1}, \ldots, X_{m}\right)$. The modules in $\mathcal{G}$ belong to $\mathcal{T}\left(X_{1}, \ldots, X_{m}\right)$ and $X_{1}, \ldots, X_{m}$ belong to $\mathcal{G}$. Thus, it is sufficient to show that $\mathcal{G}$ is a torsion class.

Since $\mathcal{G}$ is the filtered union of classes closed under epimorphisms, it is closed under epimorphisms. In order to show that $\mathcal{G}$ is closed under extensions, we follow the proof for the first assertion of Proposition 1 as closely as possible: Let $g^{\prime}: F^{\prime} \rightarrow M^{\prime}$ and $g^{\prime \prime}: F^{\prime \prime} \rightarrow$ $M^{\prime \prime}$ be surjective maps with $F^{\prime}, F^{\prime \prime}$ in add $\mathcal{F}_{s}$ for some $s$. Let $0 \rightarrow M^{\prime} \rightarrow M \rightarrow M^{\prime \prime} \rightarrow 0$ be an exact sequence. The induced exact sequence with respect to $g^{\prime \prime}$ is of the form $0 \rightarrow M^{\prime} \rightarrow Y_{1} \rightarrow F^{\prime \prime} \rightarrow 0$ with a surjective map $g_{1}: Y_{1} \rightarrow M$. Since $\Lambda$ is hereditary and 
$g^{\prime}$ is surjective, there is an exact sequence $0 \rightarrow F^{\prime} \rightarrow Y_{2} \rightarrow F^{\prime \prime} \rightarrow 0$ with a surjective map $g_{2}: Y_{2} \rightarrow Y_{1}$. Since $F^{\prime}, F^{\prime \prime}$ belong to $\mathcal{F}$ and their (relative) Loewy length is at most $s$, the exact sequence shows that $M$ also belongs to $\mathcal{F}$ and has (relative) Loewy length at most $2 s$. The surjective map $g_{1} g_{2}: Y_{2} \rightarrow M$ shows that $M$ is in $\mathcal{G}\left(\mathcal{F}_{2 s}\right) \subseteq \mathcal{G}$.

Now assume that $C$ is a cover for $\mathcal{G}$. The module $C$ belongs to $\mathcal{G}\left(\mathcal{F}_{r}\right)$ for some $r$, thus there is an epimorphism $f: F \rightarrow C$ for some $F \in \mathcal{F}_{r}$. With $C$ also $F$ is a cover for $\mathcal{G}$. Note that there is a module $F^{\prime}$ which belongs to $\mathcal{F}_{r+1}$ and not to $\mathcal{F}_{r}$, for example any object in $\mathcal{F}$ which is (relative) serial and has (relative) length equal to $r+1$. Since $F^{\prime}$ is in $\mathcal{G}$, and $F$ is a cover of $\mathcal{G}$, the module $F^{\prime}$ is generated by $F$. But if $F^{\prime}$ is generated by $F$, its (relative) Loewy length is at most $r$. This means that $F^{\prime}$ is in $\mathcal{F}_{r}$, a contradiction.

\section{Construction of Ext-pairs.}

Proposition 3. A connected hereditary artin algebra which is representation-infinite and has at least three simple modules has Ext-pairs.

Given a finite dimensional algebra $R$, we denote by $Q(R)$ its Ext-quiver: its vertices are the isomorphism classes $[S]$ of the simple $R$-modules $S$, and given two simple $R$-modules $S, S^{\prime}$, there is an arrow $[S] \rightarrow\left[S^{\prime}\right]$ provided $\operatorname{Ext}^{1}\left(S, S^{\prime}\right) \neq 0$. If $R$ is hereditary, then clearly $Q(R)$ is directed. If necessary, we endow $Q(R)$ with a valuation as follows: Given an arrow $S \rightarrow S^{\prime}$, consider $\operatorname{Ext}\left(S, S^{\prime}\right)$ as a left $\operatorname{End}(S)^{\mathrm{op}}$-module or as a left $\operatorname{End}\left(S^{\prime}\right)$-module and put

$$
v\left([S],\left[S^{\prime}\right]\right)=\left(\operatorname{dim}_{\operatorname{End}(S)} \operatorname{Ext}\left(S, S^{\prime}\right)\right)\left(\operatorname{dim}_{\operatorname{End}\left(S^{\prime}\right)^{\text {op }}} \operatorname{Ext}\left(S, S^{\prime}\right)\right)
$$

(note that in contrast to [DR], we only will need the product of the two dimensions, not the pair). Given a vertex $i$ of $Q(R)$, we denote by $S(i), P(i), I(i)$ a simple, projective or injective module corresponding to the vertex $i$, respectively.

We later will use the following: If $Q(\Lambda)=(1 \rightarrow 2)$, then the arrow $1 \rightarrow 2$ has valuation at least 2 if and only if $I(2)$ is not projective if and only if $P(1)$ is not injective; if the arrow $1 \rightarrow 2$ has valuation at least 3 , then $\tau S(1)$ (where $\tau$ is the Auslander-Reiten translation) is neither projective, nor a neighbor of $P(1)$ in the Auslander-Reiten quiver, consequently $\operatorname{Hom}\left(P(1), \tau^{2} S(1)\right) \neq 0$, thus $\operatorname{Ext}(\tau S(1), P(1)) \neq 0$.

For any hereditary algebra $\Lambda$ with $Q(\Lambda)$ being a tree quiver, it is easy to construct a sincere exceptional module, using induction: If $Q^{\prime}$ is a subquiver of $Q$ such that $Q$ is obtained from $Q^{\prime}$ by adding just one vertex $\omega$ and one arrow, and $M^{\prime}$ is an exceptional module for the restriction of $\Lambda$ to $Q^{\prime}$, then let $M$ be the universal extension of $M^{\prime}$ by copies of $S(\omega)$; here we consider extensions from above or from below, provided $\omega$ is a source or a sink, respectively.

For the proof of Proposition 3, we consider four special cases:

Case 1. The algebra $\Lambda$ is tame.

We use the structure of the Auslander-Reiten quiver of $\Lambda$ as presented in [DR]. Since we assume that $\Lambda$ has at least 3 vertices, there is a tube of rank $r \geq 2$. The simple regular modules in this component form an Ext-cycle of cardinality $r$, say $X_{1}, \ldots, X_{r}$. There is a unique indecomposable module $Y$ with a filtration $Y=Y_{0} \supset Y_{1} \supset \cdots \supset Y_{r-1}=0$ such that $Y_{i-1} / Y_{i}=X_{i}$ for $1 \leq i \leq r-1$. Clearly, the pair $Y, X_{r}$ is an Ext-pair.

Case 2. The quiver $Q=Q(\Lambda)$ is not a tree. 
Deleting, if necessary, vertices, we may assume that the underlying graph of $Q$ is a cycle. Let $w$ be a path from a sink $i$ to a source $j$ of smallest length, let $Q^{\prime}$ be the subquiver of $Q$ given by the vertices and the arrows which occur in $w$. Not every vertex of $Q$ belongs to $Q^{\prime}$, since otherwise $Q$ is obtained from $Q^{\prime}$ by adding just arrows, thus by adding a unique arrow, namely an arrow $i \rightarrow j$. But then this arrow is also a path from a sink to a source, and it has length 1 . By the minimality of $w$, we see that also $w$ has length 1 and therefore $Q$ has just the two vertices $i, j$. But then $Q$ can have only one arrow, thus is a tree. This is a contradiction.

Let $Q^{\prime \prime}$ be the full subquiver given by all vertices of $Q$ which do not belong to $Q^{\prime}$. Of course, $Q^{\prime \prime}$ is connected (it is a quiver of type $\mathbb{A}$ ). Let $X$ be an exceptional module with support $Q^{\prime}$ and $Y$ an exceptional module with support $Q^{\prime \prime}$. Since $Q^{\prime}, Q^{\prime \prime}$ have no vertex in common, we see that $\operatorname{Hom}(X, Y)=0=\operatorname{Hom}(Y, X)$.

There is an arrow $i \rightarrow j^{\prime \prime}$ with $j^{\prime \prime}$ a vertex of $Q^{\prime \prime}$. This arrow shows that $\operatorname{Ext}^{1}(X, Y) \neq$ 0 . Similarly, there is an arrow $i^{\prime \prime} \rightarrow j$ with $i^{\prime \prime}$ a vertex of $Q^{\prime \prime}$. This arrow shows that $\operatorname{Ext}^{1}(Y, X) \neq 0$.

We consider now algebras $\Lambda$ with Ext-quiver $1 \rightarrow 2 \rightarrow 3$. We denote by $\Lambda^{\prime}$ the restriction of $\Lambda$ to the subquiver with vertices 1,2 , and by $\Lambda^{\prime \prime}$ the restriction of $\Lambda$ to the subquiver with vertices 2,3 . Given a representation $M$, let $M_{3}$ be the sum of all submodules of $M$ which are isomorphic to $S(3)$, then $M / M_{3}$ is a $\Lambda^{\prime}$-module.

Lemma 2. Let $X, Y$ be $\Lambda$-modules. If $X_{3}=0$ and $\operatorname{Ext}^{1}\left(Y / Y_{3}, X\right) \neq 0$, then also $\operatorname{Ext}^{1}(Y, X) \neq 0$.

Proof: The exact sequence $0 \rightarrow Y_{3} \rightarrow Y \rightarrow Y / Y_{3} \rightarrow 0$ yields an exact sequence

$$
\operatorname{Hom}\left(Y_{3}, X\right) \rightarrow \operatorname{Ext}^{1}\left(Y / Y_{3}, X\right) \rightarrow \operatorname{Ext}^{1}(Y, X)
$$

The first term is zero, since $Y_{3}$ is a sum of copies of $S(3)$ and $X_{3}=0$. Thus, the map $\operatorname{Ext}^{1}\left(Y / Y_{3}, X\right) \rightarrow \operatorname{Ext}^{1}(Y, X)$ is injective.

Case 3. $Q(\Lambda)=(1 \rightarrow 2 \rightarrow 3)$, and $v(1,2) \geq 2, v(2,3) \geq 2$.

Let $X=S(2)$ and let $Y$ be the universal extension of $X$ using the modules (1) and $S(3)$ (thus, we form the universal extension from above using copies of $S(1)$ and the universal extension from below using copies of $S(3)$. Clearly, $Y$ is exceptional. Since the socle of $Y$ consists of copies of $S(3)$, we have $\operatorname{Hom}(S(2), Y)=0$. Since the top of $Y$ consists of copies of $S(1)$, we have $\operatorname{Hom}(Y, S(2))=0$.

Since $v(1,2) \geq 2$, the module $Y / Y_{3}$ is not a projective $\Lambda^{\prime}$-module. As a consequence, $\operatorname{Ext}\left(Y / Y_{3}, S(2)\right) \neq 0$. Lemma 2 shows that also $\operatorname{Ext}(Y, S(2)) \neq 0$. By duality, we similarly see that $\operatorname{Ext}(S(2), Y) \neq 0$.

Case 4. $Q(\Lambda)=(1 \rightarrow 2 \rightarrow 3)$, and $v(1,2) \geq 3, v(2,3)=1$.

Let $X=P(1) / P(1)_{3}$ (thus $X$ is the projective $\Lambda^{\prime}$-module with top $S(1)$ ). Let $Y=\tau X$, where $\tau=D \operatorname{Tr}$ is the Auslander-Reiten translation in $\bmod \Lambda$. Of course, both modules $X, Y$ are exceptional. Since $Y=\tau X$, we know already that $\operatorname{Ext}^{1}(X, Y) \neq 0$.

We claim that $Y / Y_{3}=\tau^{\prime} S(1)$, where $\tau^{\prime}$ is the Auslander-Reiten translation of $\Lambda^{\prime}$. Since $P(1)_{3}=S(3)^{a}$ for some $a \geq 1$, a minimal projective presentation of $X$ has the form

$$
0 \rightarrow S(3)^{a} \rightarrow P(1) \rightarrow X \rightarrow 0
$$


thus the defining exact sequences for $Y=\tau X$ is of the form

$$
0 \rightarrow Y \rightarrow I(3)^{a} \rightarrow S(1) \rightarrow 0
$$

In order to obtain $\tau^{\prime} S(1)$, we start with a minimal projective presentation

$$
0 \rightarrow S(2)^{a} \rightarrow P^{\prime}(1) \rightarrow S(1) \rightarrow 0
$$

where $P^{\prime}(1)$ is the projective cover of $S(1)$ as a $\Lambda^{\prime}$-module (actually, $P^{\prime}(1)=X$ ). Since $\nu(2,3)=1$, the number $a$ in $(*)$ and $(* *)$ is the same. The defining exact sequences for $Y=\tau X$ and $\tau^{\prime} S(1)$ are part of the following commutative diagram with exact rows and columns:

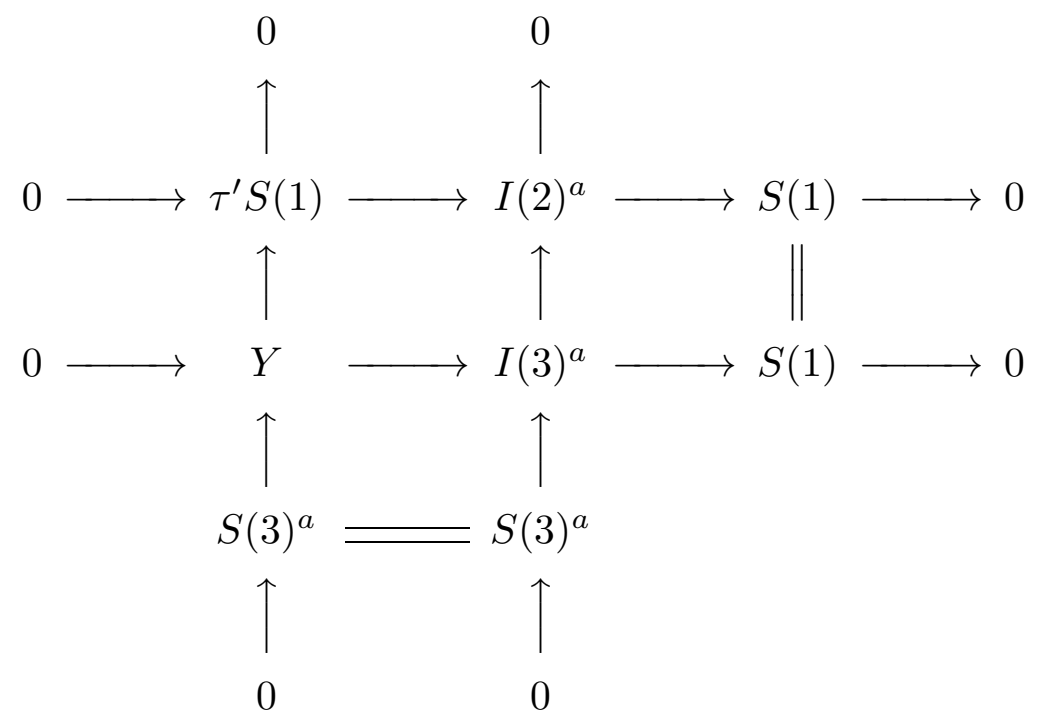

The left column shows that $Y / Y_{3}=\tau^{\prime} S(1)$.

We have noted already that $v(1,2) \geq 3$ implies that $\operatorname{Ext}\left(\tau^{\prime} S(1), P^{\prime}(1)\right) \neq 0$. According to Lemma 2, we see that $\operatorname{Ext}(Y, X) \neq 0$.

Finally, let us show that $X, Y$ are orthogonal. Any homomorphism $Y \rightarrow X$ vanishes on $Y_{3}$, since $X$ has no composition factor $S(3)$. Now $Y / Y_{3}$ is indecomposable and not projective as a $\Lambda^{\prime}$-module, whereas $X$ is a projective $\Lambda^{\prime}$-module, thus $\operatorname{Hom}(Y, X)=$ $\operatorname{Hom}\left(Y / Y_{3}, X\right)=0$.

On the other hand, the restriction $X^{\prime \prime}$ of $X$ to the subquiver $Q^{\prime \prime}$ with vertices 2,3 is a sum of copies of $S(2)$, whereas the restriction of $Y$ to the subquiver $Q^{\prime \prime}$ is a projectiveinjective module. It follows that the restriction of any homomorphism $f: X \rightarrow Y$ vanishes on $X^{\prime \prime}$. Thus $f$ factors through a direct sum of copies of $S(1)$. But $S(1)$ is injective and obviously not a submodule of $Y$. It follows that $f=0$.

Remark. Concerning the cases 3 and 4 , there is an alternative proof which uses dimension vectors and the Euler form on the Grothendieck group $K_{0}(\Lambda)$. But for this approach, one needs to deal with the valuation of $Q(\Lambda)$ as in $[\mathrm{DR}]$, attaching to any arrow $i \rightarrow j$ a pair $(a, b)$ of positive numbers instead of the single number $v(i, j)=a b$.

Proof of Proposition 3. Let $\Lambda$ be connected, hereditary, representation-infinite, with at least 3 simple modules. Case 2 shows that we can assume that $Q(\Lambda)$ is a tree. Assume that there is a subquiver $Q^{\prime}$ such that at least two of the arrows have valuation at least 2 , 
choose such a $Q^{\prime}$ of minimal length. We want to construct an Ext-pair for the restriction of $\Lambda$ to $Q^{\prime}$. Using reflection functors (see [DR]), we can assume that $Q^{\prime}$ has orientation $1 \rightarrow 2 \rightarrow \cdots \rightarrow n-1 \rightarrow n$. If $n=3$, then this is case 3 . Thus assume $n \geq 4$. The minimality of $Q^{\prime}$ asserts that $\nu(i, i+1)=1$ for $2 \leq i \leq n-2$. If we denote by $\Lambda^{\prime}$ the restriction of $\Lambda$ to $Q^{\prime}$, then $\Lambda^{\prime}$ has a full exact abelian subcategory $\mathcal{U}$ which is equivalent to the module category of an algebra as discussed in case 3 (namely the subcategory of all $\Lambda^{\prime}$-modules which do not have submodules of the form $S(i)$ with $2 \leq i \leq n-2$ and no factor modules of the form $S(i)$ with $3 \leq i \leq n-1)$. Since $\mathcal{U}$ has Ext-pairs, also mod $\Lambda$ has Ext-pairs. Thus, we can assume that at most one arrow $i \rightarrow j$ has valuation greater than 2 . If $v(i, j) \geq 3$, then we take a connected subquiver $Q^{\prime}$ with 3 vertices containing this arrow $i \rightarrow j$. If necessary, we use again reflection functors in order to change the orientation so that we are in case 4 . Thus we are left with the representation-infinite algebras $\Lambda$ with the following properties: $Q(\Lambda)$ is a tree, there is no arrow with valuation greater than 2 and at most one arrow with valuation equal to 2 . It is easy to see that $Q(\Lambda)$ contains a subquiver $Q^{\prime}$ such that the restriction of $\Lambda$ to $Q^{\prime}$ is tame, thus we can use case 1 .

Proof of Theorem. Let $\Lambda$ be connected and hereditary. If $\Lambda$ is representation-finite, then tors $\Lambda=$ f-tors $\Lambda$, thus f-tors $\Lambda$ is a lattice. If $\Lambda$ has precisely two simple modules, then f-tors $\Lambda$ can be described easily (see the proof of Proposition 2.2 in [IRTT] which works in general), it obviously is a lattice.

On the other hand, if $\Lambda$ is representation-infinite and has at least three simple modules, then Proposition 3 asserts that $\Lambda$ has an Ext-pair, say $X, Y$. Since $X, Y$ are exceptional modules, Proposition 1 shows that $\mathcal{T}(X)=\mathcal{G}(X)$ and $\mathcal{T}(Y)=\mathcal{G}(Y)$ both belong to f-tors $\Lambda$. The join of $\mathcal{T}(X)$ and $\mathcal{T}(Y)$ in tors $\Lambda$ is $\mathcal{T}(X, Y)$. According to Proposition 2, $\mathcal{T}(X, Y)$ does not belong to f-tors $\Lambda$.

\section{References}

[AS] M. Auslander, S. O. Smalø: Preprojective modules over artin algebras, J. Algebra 66 (1980) 61-122.

[DR] V. Dlab, C. M. Ringel: Indecomposable representations of graphs and algebras. Mem. Amer. Math. Soc. 173 (1976).

[IRTT] O. Iyama, I. Reiten, H. Thomas, G. Todorov: Lattice structure of torsion classes for path algebras of quivers. arXiv:1312.3659

[R] C. M. Ringel: Representations of $k$-species and bimodules. J. Algebra 41 (1976), 269-302.

[Ro] A. V. Roiter: Unboundedness of the dimension of the indecomposable representations of an algebra which has infinitely many indecomposable representations. Izv. Akad. Nauk SSSR. Ser. Mat. 32 (1968), 1275-1282

C. M. Ringel

Department of Mathematics, Shanghai Jiao Tong University

Shanghai 200240, P. R. China, and

King Abdulaziz University, P O Box 80200

Jeddah, Saudi Arabia

e-mail: ringel@math.uni-bielefeld.de 